\title{
HCN observations of comets C/2013 R1 (Lovejoy) and C/2014 Q2 (Lovejoy) (Research Note)
}

\author{
E. S. Wirström ${ }^{1}$, M. S. Lerner ${ }^{1}$, P. Källström ${ }^{2}$, A. Levinsson ${ }^{3}$, A. Olivefors ${ }^{2}$, and E. Tegehall ${ }^{2}$ \\ 1 Department of Earth and Space Sciences, Chalmers University of Technology, Onsala Space Observatory, 43992 Onsala, Sweden \\ e-mail: eva.wirstrom@chalmers.se \\ 2 Department of Chemistry and Chemical Engineering, Chalmers University of Technology, 41296 Gothenburg, Sweden \\ 3 Department of Applied Physics, Chalmers University of Technology, 41296 Gothenburg, Sweden
}

Received 30 September 2015 / Accepted 18 February 2016

\begin{abstract}
HCN $J=1-0$ emission from the long-period comet C/2013 R1 (Lovejoy) was observed from the Onsala Space Observatory on multiple occasions during the month before its perihelion passage on December 22, 2013. We report detections for seven different dates, spanning heliocentric distances $\left(R_{h}\right)$ decreasing from 0.94 to 0.82 au. Estimated HCN production rates are generally higher than previously reported for the same time period, but the implied increase in production rate with heliocentric distance, $Q_{\mathrm{HCN}} \propto R_{h}^{-3.2}$, represent well the overall documented increase since it was first observed at $R_{\mathrm{h}}=1.35$. The implied mean $\mathrm{HCN}$ abundance relative to water in $\mathrm{R} 1$ Lovejoy is $0.2 \%$. We also report on a detection of $\mathrm{HCN}$ with the new $3 \mathrm{~mm}$ receiver system at Onsala Space Observatory in comet C/2014 Q2 (Lovejoy) on January 14, 2015, when its heliocentric distance was $1.3 \mathrm{au}$. Relative to comet C/2013 R1 (Lovejoy), the $\mathrm{HCN}$ production rate of $\mathrm{C} / 2014 \mathrm{Q} 2$ (Lovejoy) was more than 5 times higher at similar heliocentric distances, and the implied $\mathrm{HCN}$ abundance relative to water $0.09 \%$.
\end{abstract}

Key words. comets: individual: C/2013 R1 (Lovejoy) - comets: individual: C/2014 Q2 (Lovejoy) - radio lines: planetary systems comets: general

\section{Introduction}

Comets, bodies composed of molecular ices and crystalline silicate dust, were formed far out from the Sun in the young solar system, and the vast majority have remained there ever since. Their compositions can therefore provide clues to the local conditions during the formation of the solar system (Mumma \& Charnley 2011; Bockelée-Morvan et al. 2004). When a cometary orbit is perturbed, bringing it within about 3 au from the Sun, the ices in its nucleus sublimate to form a coma of gaseous volatiles and dust, allowing for remote investigations of their composition. Spectroscopic investigations at infrared (e.g. DiSanti et al. 2013) and radio wavelengths (e.g. Remijan et al. 2008; Biver et al. 2012) have revealed a wide chemical diversity among comets. Thus, detailed studies of cometary comae are essential to explore the extent of heterogeneity amongst comets, and provide further understanding of the distribution of molecular material throughout the solar system during its formation.

The water production rate of a comet is expected to scale approximately as $R_{\mathrm{h}}^{-2.0}$, where $R_{\mathrm{h}}$ is the comet's radial distance to the Sun. HCN is one of the cometary molecules whose production rate follows most closely that of water, typically with a mixing ratio close to $0.1 \%$; therefore, this molecule is commonly used as a proxy to the water production rate (Mumma \& Charnley 2011). By combining observations of HCN over a range of heliocentric distances, the production rate variability can be studied and possibly connected to outgassing mechanisms, physical structure, and distribution of volatiles near the nucleus surface.
We report on observations of hydrogen cyanide $(\mathrm{HCN})$ in comet C/2013 R1 (Lovejoy) (hereafter referred to as R1 Lovejoy) and C/2014 Q2 (Lovejoy) (hereafter Q2 Lovejoy). Comet R1 Lovejoy is a high-inclination $\left(i=64^{\circ}\right)$, long-period comet with orbital period of about $7000 \mathrm{yr}$. It was discovered by Terry Lovejoy on September 7, 2013 at a heliocentric distance of 1.98 au (Lovejoy et al. 2013), and passed perihelion at 0.81 au on December 22, 2013. Comet Q2 Lovejoy was also discovered by Terry Lovejoy, almost a year later on August 17, 2014 (Lovejoy et al. 2014), at a heliocentric distance of 2.63 au. Comet Q2 Lovejoy is also a long-period, high-inclination $\left(i \sim 80^{\circ}\right)$ comet that reached perihelion on January 30, 2015, at a closest heliocentric distance of $1.29 \mathrm{au}$.

\section{Observations and data reduction}

The $J=1-0$ transition of $\mathrm{HCN}$ at $88.63 \mathrm{GHz}$ was observed towards comet C/2013 R1 (Lovejoy) using the $3 \mathrm{~mm}$ receiver at the Onsala Space Observatory $20 \mathrm{~m}$ antenna (OSO20 m) on multiple occasions during November and December 2013. At the time of observations, comet R1 Lovejoy was approaching perihelion, but receding from Earth. The geometric circumstances for the observing dates are included in Table 1.

A frequency switching mode with a switching frequency of $5 \mathrm{~Hz}$ and a throw of $5 \mathrm{MHz}$ was used, and spectra were recorded with a 1600 channel correlator at channel separation $12.5 \mathrm{kHz}$, thus providing $20 \mathrm{MHz}$ bandwidth. Before each observing session, tabulated hourly ephemerides from the latest available solution were obtained from the Jet Propulsion 
Table 1. Summary of observations and results.

\begin{tabular}{|c|c|c|c|c|c|c|c|c|}
\hline Date & $\begin{array}{c}R_{\mathrm{h}} \\
(\mathrm{au})\end{array}$ & $\begin{array}{c}\Delta \\
(\mathrm{au})\end{array}$ & $\begin{array}{l}t_{\text {int }} \\
(\mathrm{h})\end{array}$ & $\underset{\left(\mathrm{mKK} \mathrm{km} \mathrm{s}^{-1}\right)}{\int T_{\mathrm{mb}} \mathrm{d} v}$ & $\begin{array}{l}T_{\mathrm{K}} \\
(\mathrm{K})\end{array}$ & $\begin{array}{c}Q_{\mathrm{H}_{2} \mathrm{O}} \\
\left(10^{29} \mathrm{~s}^{-1}\right)\end{array}$ & $\begin{array}{c}N_{\mathrm{HCN}} \\
\left(10^{11} \mathrm{~cm}^{-2}\right)\end{array}$ & $\underset{\left(10^{26} \mathrm{~s}^{-1}\right)}{Q_{\mathrm{HCN}}}$ \\
\hline 2013 & \multicolumn{8}{|c|}{ R1 Lovejoy } \\
\hline 22 Nov. & 0.986 & 0.404 & 4.4 & $213 \pm 32$ & $62^{a}$ & $0.7^{b}$ & $8.38 \pm 1.26$ & $1.9 \pm 0.3$ \\
\hline 23 Nov. & 0.980 & 0.409 & 12.1 & $74 \pm 15$ & $62^{a}$ & $0.7^{b}$ & $2.92 \pm 0.58$ & $0.7 \pm 0.1$ \\
\hline 24 Nov. & 0.971 & 0.415 & 11.3 & $158 \pm 13$ & $63^{a}$ & $0.7^{b}$ & $6.21 \pm 0.52$ & $1.5 \pm 0.1$ \\
\hline 30 Nov. & 0.915 & 0.483 & 7.3 & $149 \pm 24$ & $66^{a}$ & $0.8^{b}$ & $5.93 \pm 0.96$ & $1.7 \pm 0.3$ \\
\hline 1 Dec. & 0.906 & 0.499 & 7.4 & $196 \pm 21$ & $67^{a}$ & $0.8^{b}$ & $7.78 \pm 0.82$ & $2.4 \pm 0.3$ \\
\hline 2 Dec. & 0.898 & 0.514 & 10.4 & $111 \pm 18$ & $69^{a}$ & $0.9^{b}$ & $4.50 \pm 0.74$ & $1.4 \pm 0.2$ \\
\hline 15 Dec. & 0.823 & 0.763 & 6.8 & $100 \pm 28$ & $81^{a}$ & $1.1^{b}$ & $3.97 \pm 1.12$ & $2.2 \pm 0.6$ \\
\hline 2015 & \multicolumn{8}{|c|}{ Q2 Lovejoy } \\
\hline 14 Jan. & 1.312 & 0.503 & 6.3 & $432 \pm 13$ & 73 & 5 & $21.3 \pm 0.7$ & $4.5 \pm 0.1$ \\
\hline
\end{tabular}

Notes. ${ }^{(a)}$ From extrapolation of $T_{\mathrm{K}}$ measurements by Biver et al. (2014), see text for details. ${ }^{(b)}$ From extrapolation of $Q_{\mathrm{H}_{2} \mathrm{O}}$ values from Biver et al. (2014).

Laboratory Horizons system ${ }^{1}$, which were then interpolated by the telescope control system. The pointing accuracy was typically checked after sunrise and sunset, and its magnitude was not found to vary significantly between observing days. Pointing offsets of less than $5^{\prime \prime}$ were found and applied to the pointing model, which has an rms of $3^{\prime \prime}$. The system temperature varied from $300 \mathrm{~K}$ and up during and between the observing days; we excluded spectra with system temperatures above $1000 \mathrm{~K}$ from the data set. The total integration times for spectra included in the analysis for each day are given in Table 1. Baselines of polynomial order 3,5 , or 7 were fitted to individual spectra and subtracted before averaging spectra using the recorded system temperatures as weights.

The same transition of $\mathrm{HCN}$ was observed toward comet C/2014 Q2 (Lovejoy) on January 14, 2015, but with the new dual-polarisation, sideband-separating $3 \mathrm{~mm}$ receiver system ${ }^{2}$ (Belitsky et al. 2015) in dual beam-switching mode (DBSW) with secondary beam offset $11^{\prime}$. The system temperature varied between 140-400 K and spectra were recorded with a fast Fourier transform spectrometer at channel separation $12.2 \mathrm{kHz}$, covering $100 \mathrm{MHz}$ bandwidth. The use of DBSW, together with the fact that the new system is more stable, made it sufficient to fit and subtract linear baselines from individual spectra before averaging with the same method as for R1 Lovejoy.

At $88.6 \mathrm{GHz}$ the OSO20 $\mathrm{m}$ beam full width at half maximum (FWHM) is about 44" corresponding to about $1.3-2.5 \times 10^{4} \mathrm{~km}$ at the distance of comet R1 Lovejoy and $1.6 \times 10^{4} \mathrm{~km}$ at the distance of Q2 Lovejoy during observations. The main beam efficiency is about 0.53 at this frequency. Before analysis and presentation, reduced spectra were redressed to a channel spacing of $0.2 \mathrm{~km} \mathrm{~s}^{-1}$.

\section{Results and analysis}

\subsection{R1 Lovejoy}

The main beam intensities, integrated over all three HCN hyperfine components and with associated $1 \sigma$ errors, are given in Table 1 for each observing day, and Fig. 1 shows the total

\footnotetext{
1 http://ssd.jpl.nasa.gov/horizons.cgi

2 http://www.chalmers.se/en/centres/oso/

radio-astronomy/20m/Pages/Description . aspx
}

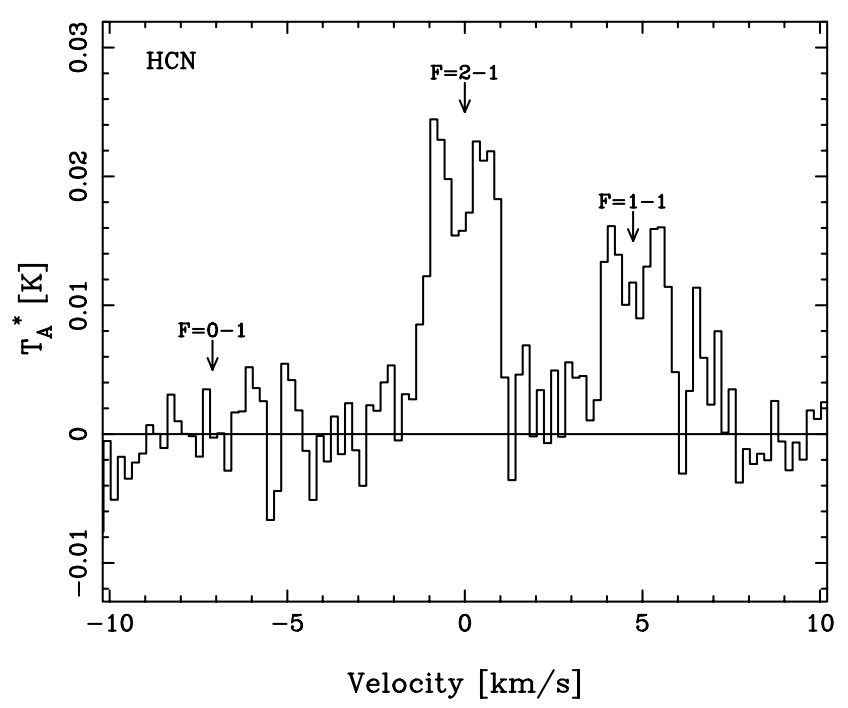

Fig. 1. HCN total average spectrum for C/2013 R1 (Lovejoy), including data from all detection days. The three hyperfine components are indicated with arrows.

average R1 Lovejoy HCN spectrum including data from all of the observing dates. The two strongest hyperfine components show clear double peaks. However, the relative intensities are fairly similar so no conclusions can be drawn about outgassing asymmetries.

No temperature information can be derived from the current dataset. However, based on observations of a set of methanol rotational transitions around $251 \mathrm{GHz}$, Biver et al. (2014) report on the coma kinetic temperatures for R1 Lovejoy on November 8 (55 K), November 27 (65 K), and December 9 (80 K), 2013. These data from concurrent observations at a similar frequency, thus probing the same part of the coma, should provide appropriate estimates of the excitation conditions for HCN. As the relation between the three reported temperatures are not at all well described by a power law, the coma kinetic temperatures on the dates of observations are approximated by linear interpolations between adjacent data points; see Table 1 . The water production rates, $Q\left(\mathrm{H}_{2} \mathrm{O}\right)$, presented in Table 1 are derived, using the same method, from the published water production rates in Biver et al. (2014). Spectroscopic parameters for HCN in its 
vibrational ground state are adopted from the Cologne Database for Molecular Spectroscopy ${ }^{3}$ (Müller et al. 2005), and the HCN $1-0$ emission is assumed to be optically thin, as verified by the ratio of line intensities.

\subsubsection{Column densities and production rates}

The rotational excitation of molecules is dominated by collisions in the inner parts of the coma, but with increasing distance from the nucleus, densities decline and radiative processes become more important. For HCN, the excitation cannot be assumed to be thermalised beyond radii of $5 \times 10^{3} \mathrm{~km}$ (Bockelée-Morvan et al. 2004); this means that local thermodynamical equilibrium (LTE) analysis of the current observations, sampling coma gas out to $\sim 10^{4} \mathrm{~km}$, is inadequate.

Therefore, to estimate the $\mathrm{HCN}$ production rate, $Q_{\mathrm{HCN}}$, and beam averaged column densities, we used the excitation and radiative transfer model described in Biver et al. (1999). The model assumes a spherically symmetric Haser model for the density distribution of molecules in the coma, and considers collisional excitation by water and electrons, as well as radiative excitation. It assumes a density distribution of parent molecules in the cometary coma that is proportional to $Q \mathrm{e}^{-r /\left(v_{\exp } t_{1}\right)} /\left(v_{\exp } r^{2}\right)$, where $v_{\text {exp }}$ is the constant gas expansion velocity and $t_{1}$ is the lifetime of the molecule. In this model, the excitation ranges from collision domination in the inner part of the coma, to domination by the balance between solar pumping and spontaneous decay at large enough radii. For more details, for example, on collision rates and excitation models that were used, see Biver et al. (1999) and references therein.

The gas expansion velocity is expected to increase as the comet approaches the Sun, but no such trend could be securely determined from our dataset. Instead, it was taken as constant and estimated from the line profiles in the total averaged spectrum shown in Fig. 1, namely as the average of the half-widths at half-maximum intensity (HWHM) for the two strongest hyperfine line components, giving $v_{\exp }=1.1 \pm 0.1 \mathrm{~km} \mathrm{~s}^{-1}$. Since HCN lifetimes are sensitive to solar activity (Bockelee-Morvan \& Crovisier 1985), and the observations were carried out at a time of moderate solar activity, a photodissociation rate of $2.0 \times 10^{-5} \mathrm{~s}^{-1}$ was adopted based on Crovisier (1994), following the example of Biver et al. (2000). This rate was scaled based on the average heliocentric distance for each observing day. The resulting HCN production rates fall between 0.7 and $2.4 \times 10^{26}$ molecules per second and are listed in the rightmost column of Table 1 .

\subsection{Q2 Lovejoy}

Figure 2 shows the total averaged $\mathrm{HCN}$ spectrum toward Q2 Lovejoy and the resulting integrated intensity is included in Table 1. The signal is stronger than for any of the dates R1 Lovejoy was observed; all three hyperfine components are clearly detected at the expected line ratios of 1:5:3. Moreover, the measured integrated intensity is more than twice what we measure for R1 Lovejoy at any date, although Q2 Lovejoy at the time of observation was further from both the Sun and the Earth. The noise level reached in just above six hours on-source time with the new receiver system is similar to what was obtained from more than 10 hours of on-source time for R1 Lovejoy in 2013.

\footnotetext{
http://www.ph1.uni-koeln.de/vorhersagen/
}

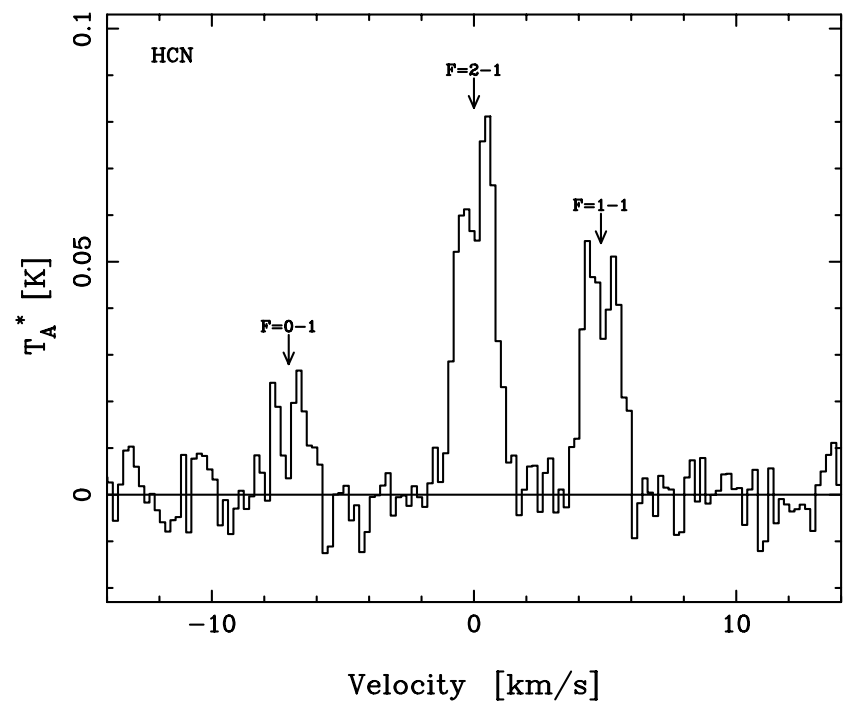

Fig. 2. Average HCN spectrum for C/2014 Q2 (Lovejoy) on January 14, 2015. The three hyperfine components are indicated with arrows.

From observations of a suite of methanol lines between January 13-26 2015, Biver et al. (2015) derive a gas kinetic temperature of $73 \mathrm{~K}$ for the coma of comet Q2 Lovejoy. The study also provides a water production rate of $5 \times 10^{29}$ molecules s $^{-1}$ for January 13-16, 2015. Adopting an expansion velocity of $0.82 \pm 0.04 \mathrm{~km} \mathrm{~s}^{-1}$ from the line profile, the $\mathrm{HCN}$ production rate is then estimated at $4.5 \times 10^{26}$ molecules per second (see Table 1) using the same model as for R1 Lovejoy.

\section{Discussion}

While the chemical reactions taking place in a cometary coma can play an important role in the radial distribution of molecules (Rodgers \& Charnley 2005), HCN is considered mainly a parent species, originating from the nucleus itself. Recent Atacama Large Millimiter Array (ALMA) spectral maps of the HCN distribution in two cometary comae are indeed consistent with centrally peaked, symmetric distributions (Cordiner et al. 2014), further supporting its designation as a parent species. This, together with the observed double-peaked line profiles, to some extent validates the use of the homogeneously expanding Haser model for estimating the $\mathrm{HCN}$ production rates presented here.

\subsection{R1 Lovejoy}

Figure 3 shows our estimated $\mathrm{HCN}$ production rates in relation to previously published rates, as a function of heliocentric distance. Our production rates are consistent with an increase in activity with decreasing heliocentric distance, best fit by the powerlaw function $Q_{\mathrm{HCN}}=1.23 \times 10^{26} \times R_{\mathrm{h}}^{-3.2} \mathrm{~s}^{-1}$, shown as a solid line in Fig. 3. Compared to production rates reported by Biver et al. (2014) from the same period of time (the errorbars of the Biver et al. dataset are too small to display at the scale of the plot), current rates are in general found to be higher.

The production rate variability implied by the current dataset has an exponential increase, $R_{\mathrm{h}}^{-3.2}$, that is steeper than what is typical for comets $\left(R_{\mathrm{h}}^{-2.0}\right.$, Biver et al. 1999). For comparison, the dash-dotted line in Fig. 3 represents a production rate variation $Q_{\mathrm{HCN}}=Q_{\mathrm{HCN}}(1 \mathrm{au}) R_{\mathrm{h}}^{-2.0}$, with a value $Q_{\mathrm{HCN}}(1 \mathrm{au})=$ $1.08 \times 10^{26} \mathrm{~s}^{-1}$ chosen to fit the first of the Paganini et al. (2014) data points. This trend fits the Biver et al. (2014) data rather well. 


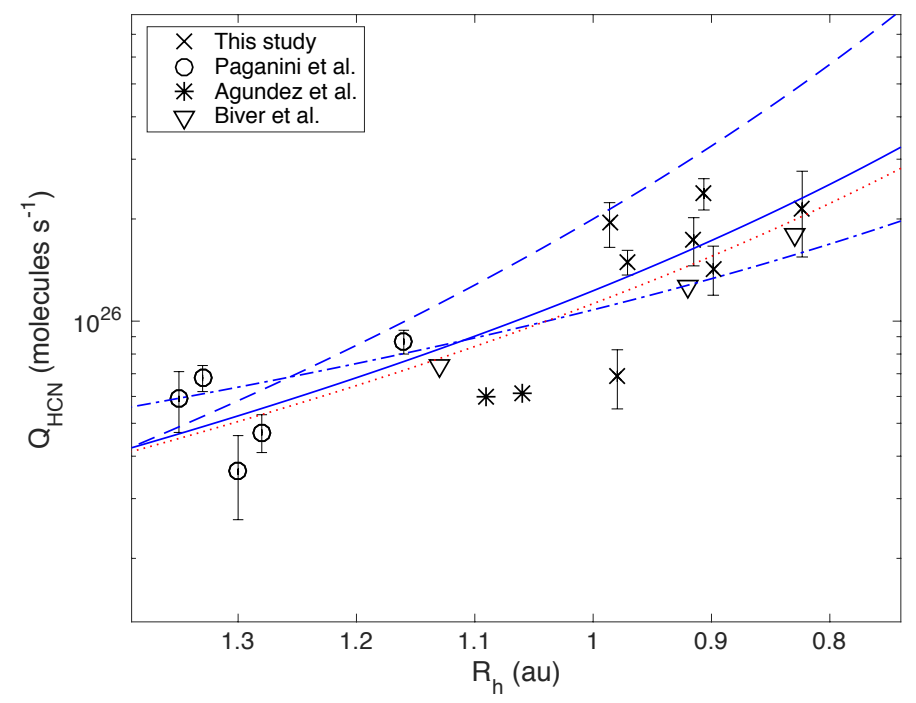

Fig. 3. HCN production rates as a function of heliocentric distance for comet C/2013 R1 Lovejoy. Error bars shown for the current data (crosses) do not include uncertainties in model parameters, but only reflect measurement errors. Circles represent data from Paganini et al. (2014), stars data from Agúndez et al. (2014), and triangles data from Biver et al. (2014). The solid line illustrates the best fit to the current data, $Q_{\mathrm{HCN}}=1.23 \times 10^{26} \times R_{\mathrm{h}}^{-3.2} \mathrm{~s}^{-1}$, while the dashed line represents $Q_{\mathrm{HCN}} \propto R_{\mathrm{h}}^{-4.7}$, the dash-dotted line $Q_{\mathrm{HCN}} \propto R_{\mathrm{h}}^{-2.0}$, and the dotted line $Q_{\mathrm{HCN}} \propto R_{\mathrm{h}}^{-3.0}$; see text for details.

On the other hand, based on their near-infrared data, Paganini et al. (2014) report an even steeper increase in the water production rate of $Q_{\mathrm{H}_{2} \mathrm{O}}=6.66 \times 10^{28} \times R_{\mathrm{h}}^{-4.7} \mathrm{~s}^{-1}$, and an HCN abundance of around $0.3 \%$ with respect to water. The dashed line in Fig. 3 represents such a production rate variation for $\mathrm{HCN}$ with $Q_{\mathrm{HCN}}(1 \mathrm{au})=0.003 \times 6.66 \times 10^{28}=2.00 \times 10^{26} \mathrm{~s}^{-1}$. We note that all production rates reported from later dates, including those of the present study, fall below this predicted trend. In fact, when assigning equal weight to all of the data points included in Fig. 3, the power law that best reproduces the $\mathrm{HCN}$ production rate variability of R1 Lovejoy is $Q_{\mathrm{HCN}}=1.13 \times 10^{26} \times R_{\mathrm{h}}^{-3.0} \mathrm{~s}^{-1}$ (dotted line in Fig. 3); this production rate variability is very similar to that derived from the current dataset alone.

Thus, even though the derived HCN production rates vary by factors of a few over timescales of days, the trend these production rates represent follows the general variation of the comet. We cannot determine whether the substantial variation is an effect of a rotating, inhomogeneous nucleus, or overall fluctuation in nucleus activity. We cannot discern any periodicity either in the variations presented here or over subsets of data from days with longer integration times. We note that the Paganini data also show substantial variation over days, albeit within a factor of two.

This study implies a global $\mathrm{HCN}$ abundance relative to water in R1 Lovejoy of $1.0-2.8 \times 10^{-3}$ with a mean of $0.2 \%$, which is somewhat lower than reported by Paganini et al. (2014) (0.3\%), but within the range of what is measured for comets in general (0.09-0.5\%; Mumma \& Charnley 2011).

\subsection{Q2 Lovejoy}

The HCN production rate of comet Q2 Lovejoy on the single day of observations was higher than what we observe from R1 Lovejoy over three weeks approaching perihelion. More noteworthy is that, compared to the production rates of $\mathrm{R} 1$ Lovejoy at similar heliocentric distances of around $1.3 \mathrm{au}$ that were determined by Paganini et al. (2014), Q2 Lovejoy was producing more than five times more $\mathrm{HCN}$; this indicates a significantly higher level of volatile outgassing from the nucleus. Corroborating this, Biver et al. (2015) concluded that Q2 Lovejoy was one of the most active comets in the last two decades, enabling these authors to detect a suite of complex organic molecules in its coma.

Our observations imply a global $\mathrm{HCN}$ abundance relative to water of $9 \times 10^{-4}$, which Biver et al. (2015) also report as the average over their observing period. This demonstrates the excellent performance of the available receiver system at the OSO20 m for comet observations.

\section{Conclusions}

We have presented Onsala $20 \mathrm{~m}$ observations of $\mathrm{HCN} J=1-0$ in comets C/2013 R1 (Lovejoy) and C/2014 Q2 (Lovejoy) within a month of their respective perihelion passages. Our estimated $\mathrm{HCN}$ production rates for R1 Lovejoy between November 22 and December 15 , at $0.7-2.4 \times 10^{26}$ molecules $\mathrm{s}^{-1}$, are somewhat higher than previously reported for overlapping dates (Biver et al. 2014). However, the implied increase in the production rates with heliocentric distance, $Q_{\mathrm{HCN}} \propto R_{\mathrm{h}}^{-3.2}$, represents well the overall increase over the time R1 Lovejoy was observed, from $R_{\mathrm{h}}=1.35$ (Paganini et al. 2014) and inward. The implied mean $\mathrm{HCN}$ abundance relative to water in R1 Lovejoy is $0.2 \%$.

The HCN production rate we estimate for Q2 Lovejoy at a heliocentric distance of $1.3 \mathrm{au}, \sim 4.5 \times 10^{26}$ molecules s $^{-1}$, is more than five times higher relative to comet R1 Lovejoy at similar heliocentric distances. The implied mean $\mathrm{HCN}$ abundance relative to water, $0.09 \%$, is the same as reported by Biver et al. (2015) for an observing period including the date of our observations.

The comparison in data quality between the presented cometary observations with the OSO20 m demonstrates the high performance of the upgraded $3 \mathrm{~mm}$ receiver system and its potential for future coma monitoring of relatively bright comets.

Acknowledgements. We sincerely thank Nicolas Biver for being gracious enough to assist in modelling the non-LTE radiative transfer and whose comments greatly improved the paper. We are also grateful to Stefanie Milam for useful advice on the analysis.

\section{References}

Agúndez, M., Biver, N., Santos-Sanz, P., Bockelée-Morvan, D., \& Moreno, R. 2014, A\&A, 564, L2

Belitsky, V., Lapkin, I., Fredrixon, M., et al. 2015, A\&A, 580, A29

Biver, N., Bockelée-Morvan, D., Crovisier, J., et al. 1999, AJ, 118, 1850 Biver, N., Bockelée-Morvan, D., Crovisier, J., et al. 2000, AJ, 120, 1554

Biver, N., Crovisier, J., Bockelée-Morvan, D., et al. 2012, A\&A, 539, A68 Biver, N., Bockelée-Morvan, D., Debout, V., et al. 2014, A\&A, 566, L5 Biver, N., Bockelée-Morvan, D., Moreno, R., et al. 2015, Sci. Adv., 1, 1500863 Bockelee-Morvan, D., \& Crovisier, J. 1985, A\&A, 151, 90

Bockelée-Morvan, D., Crovisier, J., Mumma, M. J., \& Weaver, H. A. 2004, In Comets II (Tucson: University of Arizona Press), 391

Cordiner, M. A., Remijan, A. J., Boissier, J., et al. 2014, ApJ, 792, L2

Crovisier, J. 1994, J. Geophys. Res., 99, 3777

DiSanti, M. A., Bonev, B. P., Villanueva, G. L., \& Mumma, M. J. 2013, ApJ, 763,1

Lovejoy, T., Guido, E., Howes, N., et al. 2013, Central Bureau Electronic Telegrams, 3649, 1

Lovejoy, T., Jacques, C., Pimentel, E., \& Barros, J. 2014, Central Bureau Electronic Telegrams, 3934, 1

Müller, H. S. P., Schlöder, F., Stutzki, J., \& Winnewisser, G. 2005, J. Mol. Struct., 742,215

Mumma, M. J., \& Charnley, S. B. 2011, ARA\&A, 49, 471

Paganini, L., Mumma, M. J., Villanueva, G. L., et al. 2014, ApJ, 791, 122

Remijan, A. J., Milam, S. N., Womack, M., et al. 2008, ApJ, 689, 613

Rodgers, S. D., \& Charnley, S. B. 2005, MNRAS, 356, 1542 